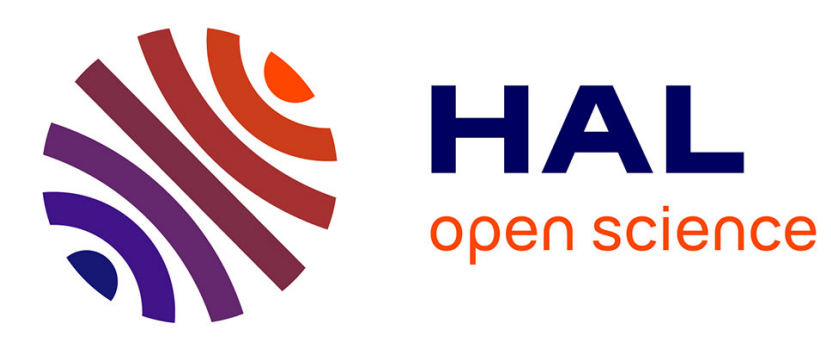

\title{
Stability of mixed-oxide titanosilicates: dependency on size and composition from nanocluster to bulk
}

\author{
Andi Cuko, Monica Calatayud, Stefan T Bromley
}

\section{To cite this version:}

Andi Cuko, Monica Calatayud, Stefan T Bromley. Stability of mixed-oxide titanosilicates: dependency on size and composition from nanocluster to bulk. Nanoscale, 2018, 10 (2), pp.832 - 842. $10.1039 / \mathrm{c} 7 \mathrm{nr} 05758 \mathrm{j}$. hal-01823256

\section{HAL Id: hal-01823256 \\ https://hal.sorbonne-universite.fr/hal-01823256}

Submitted on 25 Jun 2018

HAL is a multi-disciplinary open access archive for the deposit and dissemination of scientific research documents, whether they are published or not. The documents may come from teaching and research institutions in France or abroad, or from public or private research centers.
L'archive ouverte pluridisciplinaire HAL, est destinée au dépôt et à la diffusion de documents scientifiques de niveau recherche, publiés ou non, émanant des établissements d'enseignement et de recherche français ou étrangers, des laboratoires publics ou privés. 


\title{
Stability of Mixed-oxide Titanosilicates: Dependency on Size and Composition from Nanocluster to Bulk
}

\author{
Andi Cuko ${ }^{1,2}$, Monica Calatayud ${ }^{2,3,{ }^{*}}$, Stefan T. Bromley ${ }^{1,4, *}$ \\ ${ }^{1}$ Departament de Química Física and Institut de Química Teòrica i Computacional (IQTCUB), \\ Universitat de Barcelona, E-08028 Barcelona, Spain \\ ${ }^{2}$ Sorbonne Universités, UPMC Univ Paris 06, CNRS, Laboratoire de Chimie Théorique CC 137 - \\ 4, place Jussieu F. 75252 PARIS CEDEX 05 - France \\ ${ }^{3}$ Institut Universitaire de France, France \\ ${ }^{4}$ Institució Catalana de Recerca i Estudis Avançats (ICREA), E-08010 Barcelona, Spain
}

*Corresponding authors: s.bromley@ub.edu, calatayu@lct.jussieu.fr

Nanostructured titanosilicate materials based upon interfacing nano- $\mathrm{TiO}_{2}$ with nano- $\mathrm{SiO}_{2}$ have drawn much attention due to their huge potential for applications in a diverse range of important fields including gas sensing, (photo)catalysis, solar cells, photonics/optical components, tailored multi-(bio)functional supports and self-cleaning coatings. In each case it is the specific mixed combination of the two $\mathrm{SiO}_{2}$ and $\mathrm{TiO}_{2}$ nanophases that determines the unique properties of the final nanomaterial. In the bulk, stoichiometric mixing of $\mathrm{TiO}_{2}$ with $\mathrm{SiO}_{2}$ is limited by formation of segregated $\mathrm{TiO}_{2}$ nanoparticles or metastable glassy phases and more controlled disperse crystalline mixings only occur at small fractions of $\mathrm{TiO}_{2}(<15 \mathrm{wt} \%)$. In order to more fully understand the stability nano-SiO${ }_{2}$ and nano- $\mathrm{TiO}_{2}$ combinations with respect to composition and size, we employ accurate all-electron density functional calculations to evaluate the mixing energy in $\left(\mathrm{Ti}_{x} \mathrm{Si}_{1-x} \mathrm{O}_{2}\right)_{n}$ nanoclusters with a range of sizes $(n=2-24)$ having different titania molar fractions $(x=0-1)$. We derive all nanoclusters from a dedicated global optimisation procedure to help ensure that they are the most energetically stable structures for their size and composition. We also consider a selection of representative intimately mixed crystalline solid phase $\left(\mathrm{Ti}_{x} \mathrm{Si}_{1-\mathrm{x}} \mathrm{O}_{2}\right)_{\text {bulk }}$ systems for comparison. In agreement with experiment, we find that intimate mixing of $\mathrm{SiO}_{2}$ and $\mathrm{TiO}_{2}$ in bulk crystalline phases is energetically unfavourable. Conversely, we find that $\mathrm{SiO}_{2}-\mathrm{TiO}_{2}$ mixing is energetically favoured in small $\left(\mathrm{Ti}_{\mathrm{x}} \mathrm{Si}_{1-\mathrm{x}} \mathrm{O}_{2}\right)_{\mathrm{n}}$ nanoclusters. Following the evolution of mixing energy with nanocluster size and composition we find that mixing is most favoured in nanoclusters with a diameter of $1 \mathrm{~nm}$ with a $\mathrm{TiO}_{2}$ molar fraction of $0.3-0.4$. Thereafter, mixed nanoclusters with increasing size have progressively less negative mixing energies up to diameters of approximately $1.5 \mathrm{~nm}$. We propose some chemicalstructural principles to help rationale this energetically favourable nanoscale mixing. As a guide for 
experimentalists to observe and characterize these mixed nano-species we also provide two measurable signatures of mixing based on their unique vibrational and structural characteristics. 


\section{Introduction}

Almost all elements of the periodic table form stable oxide compounds. Due to the importance and diversity of properties exhibited by oxides (e.g. (photo)catalytic activity, multiferroic and memristive effects, superconductivity, magnetism) they play an essential role in our society in established technologies, while continuing to be a focus of frontier research in many fields. The effectiveness and range of properties of an application based on a single oxide material can often be enhanced by using mixed oxides (i.e. materials comprised by the combination of two or more oxides). Examples of such synergistic behaviour in mixed oxides are well established in, for instance, cases of catalytic activity $^{1}$ and high temperature composites ${ }^{2}$. Depending on the application, one may need to mix the constituent oxides in a more or less intimate fashion. In some cases, homogeneously mixing oxides can be difficult due to their tendency to segregate into separate phases, typically occurring when the structures and properties of the respective bulk phases are very different. Moreover, it can be also difficult to obtain specific compositions of two oxides, for instance when high temperature calcination is involved in the mixed oxide synthesis. These effects can, however, be very dependent on sample size. For example, some materials that hardly mix at bulk macroscopic level at desired composition, can be synthesized and stabilized in mixed phases at the nanoscale ${ }^{3,4,5,6}$. Furthermore, at the nanoscale, system properties can often be more finely tuned by varying both size and composition. Here, we focus on a particular mixed oxide system, titanosilicates (i.e. mixed titania $\left(\mathrm{TiO}_{2}\right)$ and silica $\left(\mathrm{SiO}_{2}\right)$ systems). Titanosilicates are robust low cost materials that can combine mechanical strength, chemical stability, nanoporosity, controllable optical characteristics, photoactivity and high redox potential properties. This combination of features make titanosilicates ideal candidates for thermally stable high surface area materials that can be tailored for a range catalytic and optical applications (e.g. industrial heterogeneous catalysis, solar cells, optical components, self-cleaning systems, selective molecular sieves, water remediation, and photocatalysis ${ }^{7,8,9,10,11,12,13}$ ). One important class of titanosilicates is that of the ETS (Engelhard Titanium Silicate) family of microporous titanosilicates introduced by Kuznicki et al. ${ }^{14,15}$. Among the numerous interesting properties of these materials, due to their tailorable pore dimensions, some are able to selectively adsorb and separate gas mixtures of small molecules with similar size, such as that of $\mathrm{N}_{2} / \mathrm{CH}_{4}, \mathrm{Ar} / \mathrm{O}_{2}$ and $\mathrm{N}_{2} / \mathrm{O}_{2}$. Formally, the titanosilicate frameworks of the ETS materials do not possess a stoichiometric mix of $\mathrm{TiO}_{2}$ and $\mathrm{SiO}_{2}$, but as with the related aluminosilicate zeolites, have a net negative charge which must be compensated by cations.

Generally, it is relatively difficult to synthesize homogeneous stoichiometric mixed $\mathrm{TiO}_{2}-\mathrm{SiO}_{2}$ bulk phases due to the tendency of the constituent oxides to segregate into two respective phases 
with increasing temperature. In crystalline systems, bulk homogeneous mixing seems to be only possible by the inclusion of a very small percentage of $\mathrm{TiO}_{2}$ in a silica framework. For higher $\mathrm{TiO}_{2}$ molar fractions homogeneous mixed $\mathrm{TiO}_{2}-\mathrm{SiO}_{2}$ oxides seem only to be possible in amorphous bulk phases, which are metastable with respect to the separate respective pure oxide polymorphs. ${ }^{16,17,18}$ There are several ways to synthesize titanosilicates. From a top down synthesis of mechanically crushing separate bulk phases of silica and titania and heating it is possible to obtain macroscopically homogeneous mixing. In order to have microscopically homogeneous mixing, however, bottom up synthetic methods such as sol-gel techniques are needed. ${ }^{19}$ When larger proportions of titania (typically > 15 wt\%) are employed these syntheses tend to lead to segregated (nano)composites of two distinct phases of $\mathrm{TiO}_{2}$ and $\mathrm{SiO}_{2}$. Dong et al. ${ }^{20}$, for example, have synthesized a highly ordered 2D hexagonal mesoporous crystalline $\mathrm{TiO}_{2}-\mathrm{SiO}_{2}$ nanocomposite with anatase $\mathrm{TiO}_{2}$ nanocrystals embedded in silica pore walls which is thermally stable at $600-850^{\circ} \mathrm{C}$. This material exhibits an improved photocatalytic activity in the degradation of rhoamine $B(\mathrm{RhB})$ compared to the commercial pure $\mathrm{TiO}_{2}$ Degussa P25 catalyst. Jiang C. et al. ${ }^{21}$ have also synthesized $\mathrm{TiO}_{2}-\mathrm{SiO}_{2}$ titanosilicate composites via sol-gel routes where $\mathrm{small}^{\mathrm{TiO}_{2}}$ anatase nanocrystals are incorporated into microporous ZSM-5 and mesoporous SBA-15 silica frameworks in order to better control their size and improve the titania photoactivity for hydrogen production by water splitting. In the class of non-segregated mixed $\mathrm{TiO}_{2}-\mathrm{SiO}_{2}$ bulk oxides, one of the most industrially important materials, known from early 80 s, is the synthetic titanosilicate- 1 (TS- 1$)^{7}$. The active sites in TS- 1 are uniformly dispersed and isolated four-fold titanium centers incorporated in a zeolitic silica MFI structure with 1.0-2.5\% of $\mathrm{TiO}_{2}$ molar inclusion. TS-1 is used as industrial redox catalyst for oxidizing organic molecules in mild conditions in the presence of hydrogen peroxide.

In this work we theoretically investigate the size-dependency of the energetics of $\mathrm{TiO}_{2}-\mathrm{SiO}_{2}$ mixing. In particular, we focus on $\mathrm{TiO}_{2}-\mathrm{SiO}_{2}$ mixing in small nanoclusters (i.e. $<100$ atoms) and how it compares with, and evolves with increasing size, towards the situation in corresponding bulk systems. In principle titanosilicate nanoparticles could be synthesised through use of ionized nanocluster beams but to the best of our knowledge no mixed silica-titania nanoclusters of the size studied herein have been reported to date. nanocluster beams are, however, becoming an important method for generating selected nanoclusters for catalytic and technological applications, ${ }^{22,23,24}$ Takeuchi et al. ${ }^{25}$, for example, have synthesized thin layers of titanosilicates by deposition of $\mathrm{TiO}_{2}$ and $\mathrm{SiO}_{2}$ nanoclusters generated by a laser beam in which they observe a high photocatalytic activity towards decomposition of $\mathrm{NO}$ in $\mathrm{N}_{2}$ and $\mathrm{O}_{2}$ due to highly disperse Ti-sites embedded in a silica framework. 
Previous theoretical studies of stoichiometric mixed $\mathrm{TiO}_{2}-\mathrm{SiO}_{2}$ systems have tended to focus on crystalline bulk mixed oxide materials having a very small fraction of $\mathrm{TiO}_{2}$ (e.g. TS-1) - see ref. 26 for an overview of theoretical and experimental studies in this area. Only very few works are present in the literature on the theoretical modelling of $\mathrm{TiO}_{2}-\mathrm{SiO}_{2}$ mixing in systems with relatively high percentages of $\mathrm{TiO}_{2}$. Recently, Landmann et al. ${ }^{27}$ reported an extensive and detailed computational modelling study of the structure of bulk amorphous titanosilicate glasses. Miroshnichenko et al. ${ }^{28}$ have studied the effect of $\mathrm{SiO}_{4}$ groups on optical, electronic, and structural properties when present in $\mathrm{TiO}_{2}$ anatase-structured nanoparticles. For very small nanoclusters, Bandyopadhyay and Aikens ${ }^{29}$ have studied a series of mixed $\mathrm{Ti}_{x} \mathrm{Si}_{y} \mathrm{O}_{2(x+y)}$ oxide nanoclusters with up to five formula units (i.e. $x+y \leq$ 5).

Confirming experiment, our calculations show that $\mathrm{TiO}_{2}-\mathrm{SiO}_{2}$ mixing is energetically unfavourable in bulk crystalline systems. However, surprisingly, in nanoclusters possessing up to 70 atoms we predict that the mixing of $\mathrm{TiO}_{2}$ and $\mathrm{SiO}_{2}$ is thermodynamically favourable. Our results point to a number of important factors which determine the higher stability of nano-titanosilicates with respect to pure nano-silica and nano-titania. Linking the results for bulk and the nanoscale we also show that the energetics of $\mathrm{TiO}_{2}-\mathrm{SiO}_{2}$ mixing depends on size; becoming less favourable with increasing size after a nanocluster size of $\sim 30$ atoms. With this work, we hope to inspire experimental groups, especially from the nanocluster beam community, to attempt the synthesis of the titanosilicate nanoclusters predicted here. To assist in the identification of mixed $\mathrm{TiO}_{2}-\mathrm{SiO}_{2}$ nanoclusters in such experiments we provide calculated vibrational frequency and average coordination number signatures that indicate when intimate nano-oxide mixing is occurring.

\section{Methodology}

We consider both mixed titanosilicates nanoclusters $\left(\mathrm{Ti}_{x} \mathrm{Si}_{1-x} \mathrm{O}_{2}\right)_{n}$, with a range of sizes $(n=2-24)$ having different titania molar fractions $(x=0-1)$, and a selection of crystalline solid phase systems $\left(\mathrm{Ti}_{x} \mathrm{Si}_{1-x} \mathrm{O}_{2}\right)_{\text {bulk. }}$ In the case of the nanoclusters, great efforts were made to try to find the most energetically stable isomer for each $\mathrm{n}$ and $\mathrm{x}$. In order to find low energy nanocluster structures on the multidimensional potential energy surface (PES) we used the Monte Carlo basin-hopping (MCBH) global optimization algorithm ${ }^{30}$ with classical interatomic potentials (IPs) to efficiently explore the PES as implemented in an in-house modified version of the GMIN 1.x code. ${ }^{31}$ Previously, IPs have been employed for global optimisation of separate $\left(\mathrm{TiO}_{2}\right)_{n}{ }^{32,33,34}$ and $\left(\mathrm{SiO}_{2}\right)_{n}^{35,36,37,38}$ nanoclusters. However, to our knowledge, IPs for mixed titanosilicate nanoclusters have not been reported. Specifically, we use the Buckingham pair interaction IP together with interionic electrostatic interactions: 


$$
V_{i j}\left(r_{i j}\right)=\frac{q_{i} q_{j}}{4 \pi \varepsilon_{0} r_{i j}}+A_{i j} \exp \left(-\frac{r_{i j}}{B_{i j}}\right)-\frac{C_{i j}}{r_{i j}^{6}}
$$

where , are ionic charges, is the vacuum dielectric constant, is the interatomic distance between atoms and and, , are fitted parameters. Due to the structural complexity of the $\left(\mathrm{Ti}_{\mathrm{x}} \mathrm{Si}_{1-\mathrm{x}} \mathrm{O}_{2}\right)_{\mathrm{n}}$ system we were not able to find one IP parameter set that could provide reliably in finding low energy nanoclusters for the full range of $x$ considered. Thus, in order to resolve this issue, we employed two different Buckingham-type IPs (IP1 and IP2) with the respective parameters reported in table 1 . These IPs are largely derived from modifications to parameters used in previously reported IPs for $\left(\mathrm{SiO}_{2}\right)_{\mathrm{n}}$ nanoclusters ${ }^{35}$ and bulk $\mathrm{TiO}_{2}{ }^{39}$. IP1 typically provides better low energy cluster isomer candidates than IP2 for systems with a higher content of $\mathrm{TiO}_{2}$ and tends to give structures where Ti-centres are 4, 5 and rarely 6 coordinated depending on nanocluster size. For systems with a lower incorporation of $\mathrm{TiO}_{2}$ this IP tends to yield 3-coordinated $\mathrm{Ti}$ atoms which are highly unstable. IP2 instead, generally yields lower energy isomer structures than IP1 for lower content of $\mathrm{TiO}_{2}$. In both cases, Si-centres tend to occupy 4-fold O-coordinated positions which are known to be to be the most energetically favourable for pure silica systems. We note that both IPs are able to provide reasonable structures for intermediate $\mathrm{TiO}_{2}$ content thus the low energy PES of this compositional range is probably most well sampled by our approach. A comparison of the performance of both IP1 and IP2 with respect to the refined energetic stabilities of the isomers coming from a typical global optimisation run over the full range of $\mathrm{TiO}_{2}$ content and for three system sizes $(N=8,910)$ is proved in the Supplementary Information.

With both IPs there is a tendency to produce nanoclusters with Si-O terminations. It is known that for small nanoclusters both pure $\mathrm{TiO}_{2}$ and $\mathrm{SiO}_{2}$ prefer open structures with terminating oxygen defects compared to compact fully coordinated ones ${ }^{33,36}$. However, due to the relatively higher ionicity of $\mathrm{Ti}-\mathrm{O}$ bonds, $\mathrm{Ti}-\mathrm{O}$ terminations tend to be less energetically costly compared to $\mathrm{Si}-\mathrm{O}$ terminations. Also, for relatively larger nanoclusters $(n \geq 12)$ there is a tendency for the IPs to produce some low energy nanocluster structures containing 5- or 6-fold coordinated silicon atoms which are relatively high in energy according to density DFT-based calculations (see details below). Instead of simply discarding these anomalous nanoclusters, we found that they could be converted into more reliably low energy candidate nanocluster structures after the MCBH search by simple interchange of cations within the respective nanocluster following: 1) Si centres in terminating Si-O defects with a 4-coordinated Ti atoms, 2.) Highly coordinated Si centres (5- and 6-coordinated) with 4-coordinated Ti centres. 


\begin{tabular}{cccc|ccc}
\hline & \multicolumn{3}{c|}{$\mathrm{IP1}\left(\mathrm{x}>0.30 \mathrm{TiO}_{2}\right)$} & \multicolumn{3}{c}{$\mathrm{IP2}\left(\mathrm{x}<0.30 \mathrm{TiO}_{2}\right)$} \\
\cline { 2 - 7 } & \multicolumn{3}{c|}{} & & & \\
\hline $\mathrm{Si}-\mathrm{O}$ & 10454.2 & 0.208 & 63.05 & 10386.97 & 0.210372 & 63.45 \\
$\mathrm{Ti}-\mathrm{O}$ & 2454.2 & 0.211 & -55.05 & 14719.71 & 0.200783 & 65.90 \\
$\mathrm{Si}-\mathrm{Si}$ & 79502.1 & 0.220 & 446.78 & 74316.65 & 0.220 & 446.78 \\
$\mathrm{Ti}-\mathrm{Si}$ & 69000.0 & 0.210 & 500.00 & 104167.92 & 0.220 & 446.78 \\
$\mathrm{Ti}-\mathrm{Ti}$ & 69000.0 & 0.210 & 480.59 & 74316.65 & 0.220 & 446.78 \\
$\mathrm{O}-\mathrm{O}$ & 2828.5 & 0.297 & 16.48 & 2828.5 & 0.297 & 16.48 \\
\hline
\end{tabular}

Table 1. Buckingham parameters for IPs used in the MCBH global optimization searches. lonic charges for both IPs are: $Q(\mathrm{Si}, \mathrm{Ti})=2.4$ and $\mathrm{Q}(\mathrm{O})=-1.4$

We used MCBH with both IP1 and IP2 for finding low energy nanocluster isomers. We typically use several $\mathrm{MCBH}$ runs for each nanocluster composition (up to five for the larger nanoclusters) and for each run we use a different initial structure. With increasing nanocluster size, we correspondingly increase the number of MCBH steps in each run from fifty thousand to one million. In addition to the usual random atomic displacements we also apply Ti/Si cation interchange moves in a small percentage (1 - 5\%) of the steps. From each run of this IP-based global optimization procedure, for each nanocluster composition, the forty lowest energy structures were then fully optimized employing all electron, relativistic, DFT based calculations using the FHI-AIMS code. ${ }^{40}$ Firstly, we employed the general gradient approximation $\mathrm{PBE}^{41}$ functional with a light/tier-1 basis of numerical atom-centred basis functions, to optimise the IP-optimised nanocluster structures coming directly from the global optimisations. We note that light/tier-1 basis of numerical atom-centred orbitals approximately provides results of a similar or higher quality to those obtained with valence triple-zeta plus polarization Gaussian type orbitals. Subsequently, from these forty isomers, the seven most stable isomers were further optimized using the hybrid PBEO functional ${ }^{42}$ with tight/tier1 basis. This cascade optimisation approach has been successfully used in previous studies ${ }^{33,34,37,43,44}$.

For the selected periodic $\left(\mathrm{Ti}_{x} \mathrm{Si}_{1-x} \mathrm{O}_{2}\right)_{\text {bulk }}$ crystals we performed an initial full optimization of both atom coordinates and cell lattice vectors at a PBE light/tier-1 level of theory with atom coordinates then further refined at a PBEO tight/tier-1 level also using FHI-AIMS. For these periodic calculations, depending on the unit cell size, we used an appropriate Monkhort-Pack grid of k-points, going from $7 \times 7 \times 7$ for the smallest system (pure rutile and quartz) to a $2 \times 2 \times 2$ points grid for the largest one.

In order to compare relative stabilities of a specific mixed system with respect to the pure oxides, we consider the mixing energy, $\Delta \quad$, which we take to be difference in the energy of the system with respect to the proportional sum of the energies of the two pure systems of the same 
size. Specifically, for a generic titanosilicate we refer to the chemical reaction between pure oxide systems as defined in equation 2 and the corresponding mixing energy in equation 3.

$$
\left.\begin{array}{llll} 
& (1-) & + & \rightarrow \\
\Delta & = & -[(1-) & ()^{+}
\end{array}\right]
$$

where is the $\mathrm{TiO}_{2}$ molar fraction in the nanocluster.

is the total DFT energy of the mixed system, ( ) and ( ) are energies of the respective pure silica and pure titania global minima structures. $\Delta \quad$ can be either positive, which means that the silica and titania mixing is energetically unfavourable, or negative, which means that the mixing is favourable. Mixing energies are then normalized by the number of $\mathrm{MO}_{2}(\mathrm{M}=\mathrm{Si}, \mathrm{Ti})$ oxide units. In the case of nanoclusters the number of units corresponds to its size $n$, while in the case of bulk systems we employ the number of oxide units in the unit cell. We note that normalization factor could also be chosen to be the number of the smallest titanosilicate units according to a specific composition (e.g. when $x=0.5$ the fundamental unit is $\mathrm{TiSiO}_{4}$, when $\mathrm{x}=0.333$ the fundamental unit is $\mathrm{TiSi}_{2} \mathrm{O}_{6}$ etc.). This normalisation choice unit is typically employed for reporting experimental mixing enthalpies of bulk systems. For example, conventionally, experimental mixing enthalpies of two common mixed $\mathrm{MgO}^{-\mathrm{SiO}_{2}}$ magnesium silicates forsterite $\left(\mathrm{Mg}_{2} \mathrm{SiO}_{4}\right)$ and enstatite $\left(\mathrm{MgSiO}_{3}\right)$ are usually given relative to moles of $\mathrm{Mg}_{2} \mathrm{SiO}_{4}$ and moles of $\mathrm{MgSiO}_{3}$ respectively. ${ }^{45}$ For titanosilicates, to the best of our knowledge, there are no experimental enthalpies of mixing because of the tendency of the constituent oxide phases to segregate. We note that the use of the $\mathrm{MO}_{2}(\mathrm{M}=\mathrm{Si}, \mathrm{Ti})$ oxide unit also allows us to directly compare systems with different compositions on the same footing. In the Supplementary Information we report $\Delta \quad / \quad(\mathrm{eV})$ according to both definitions of normalisation unit for a range of $\mathrm{Ti}_{0.5} \mathrm{Si}_{0.5} \mathrm{O}_{2}$ systems. We note that the mixing energy in equation 3 is a thermodynamic quantity, therefore formally all terms used should be thermally corrected at finite temperatures. As a first approximation, we mainly consider $\Delta \quad$ in terms of $\mathrm{OK}$ internal electronic energies directly coming from DFT calculations. However, in order to account for the effect of finite temperature we have also estimated the free mixing energies $(\Delta \quad)$ for a set of mixed titanosilicates nanoclusters with with $1: 1$ stoichiometry $(x=0.5)$. For these calculations we evaluated the harmonic vibrational frequencies and resulting free energies with the Gaussian09 package ${ }^{46}$ using the PBEO functional with the Ahlrichs triple- $\zeta$ plus polarizations basis functions (TZPV) $)^{47}$ on all atoms. 


\section{Results and Discussions}

\section{1) Bulk mixed $\mathrm{TiO}_{2}-\mathrm{SiO}_{2}$ systems}

Firstly we took a selection of mixed $\left(\mathrm{Ti}_{x} \mathrm{Si}_{1-\mathrm{x}} \mathrm{O}_{2}\right)_{\text {bulk }}$ crystalline systems where one or more 4-fold coordinated Si-centres were replaced with $\mathrm{Ti}$ atoms to generate mixed oxide bulk phases with different degrees of mixing (i.e. $x=0.08,0.33,0.50$ and $0.67 \mathrm{TiO}_{2}$ molar fraction). Specifically, we consider the quartz (Fig. 1a) and sodalite (Fig. 1b) crystal structure types. We choose quartz as representative of dense titanosilicates where we examined structures with $x=0.33,0.50$ and 0.67 . The sodalite crystal with low $(\mathrm{x}=0.08) \mathrm{TiO}_{2}$ inclusion is taken to represent low density zeolitic titanosilicates such as TS-1. In the latter case we did not consider TS-1 directly because of the prohibitive computational cost of PBEO DFT calculations with the large unit cell involved. In our selected set of bulk crystal titanosilicates, we also include a hypothetical phase having 4-fold Sicentres and 6-fold Ti-centres and an intermediate inclusion of $\mathrm{TiO}_{2}(\mathrm{x}=0.33$, see Fig. 1c). This hypothetical structure was found in the Materials Project database (ID: $\mathrm{mp}-766596)^{48}$. We note that unlike quartz and sodalite, this latter structure exhibits one dimensional "wires" of linked $\mathrm{TiO}_{6}$ units as found in the experimentally synthesised non-stoichiometric ETS-10 structure ${ }^{10}$. This high 6-fold coordination environment of $\mathrm{Ti}$ is also similar to that found in the most stable rutile $\mathrm{TiO}_{2}$ bulk polymorph. We refer to this structure as the "wired" phase.

a)

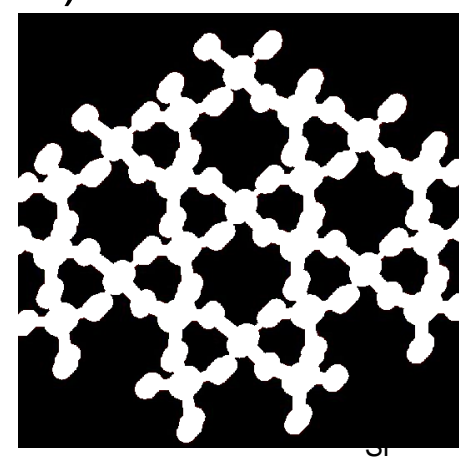

b)

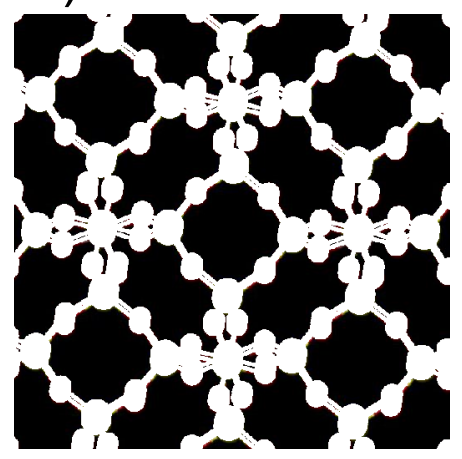

c)

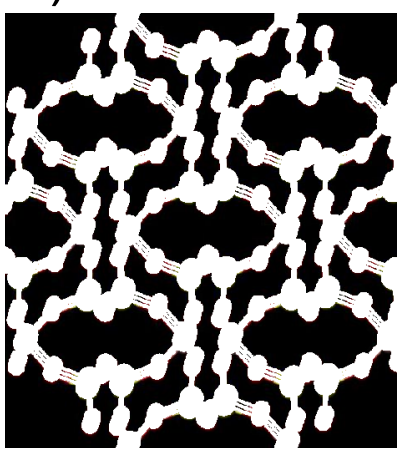

Fig 1. Crystalline mixed titanosilicates models: a) quartz-like structures (there are three quartz-like systems with different $\mathrm{TiO}_{2}$ molar fractions: $\mathrm{x}=0.33,0.50$ (shown here) and $0.67, \mathrm{~b}$ ) a sodalite-like system where in the unit cell, one Si atom is replaced with a Ti atom which corresponds to $\mathrm{x}=0.08, \mathrm{c}$ ) is the "wired" titanosilicate structure found in the Materials Project database having 4-fold silicon and 6-fold titanium atoms with $\mathrm{x}=0.33$.

\section{2) Global optimized mixed nanocluster structures}


We report a set of global optimized mixed $\left(\mathrm{Ti}_{x} \mathrm{Si}_{1-x} \mathrm{O}_{2}\right)_{n}$ titanosilicate nanoclusters with $n=2-24$ units. For $\mathrm{n}=2-10$ we consider all possible values of molar fraction $x$. We also consider some larger nanoclusters with $n=12,14,16$ and 24 , for which selected mixing ratios have been chosen.

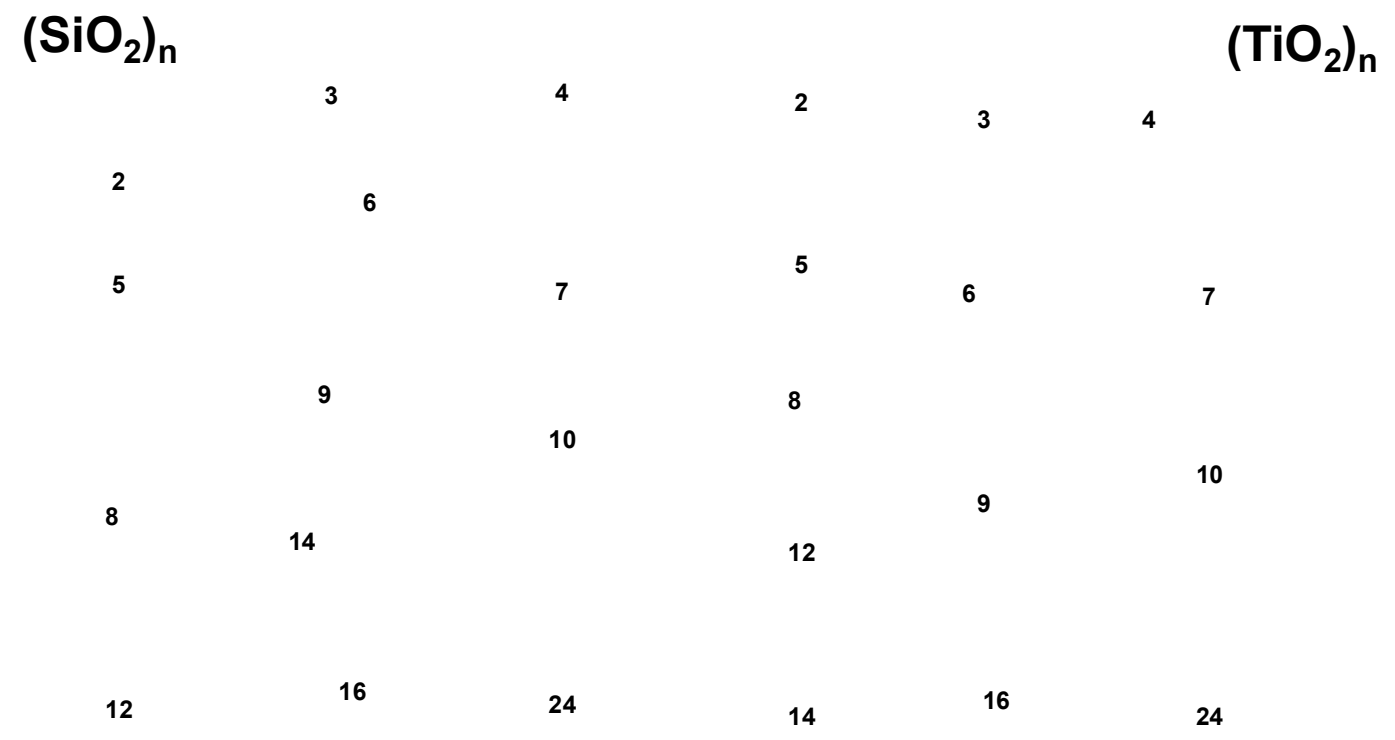

Fig 2. Structures of globally minimised nanoclusters of silica (left) and titania (right), each labelled with the respect to the corresponding number of $\mathrm{MO}_{2}(\mathrm{M}=\mathrm{Si}$ or $\mathrm{Ti})$ units. All these structures have been previously reported in the literature by us and other authors. ${ }^{32,33,34,36,37,38,49,50,51,52}$ Atom key: yellow - silicon, grey titanium, red - oxygen.

In Fig. 2 we show the lowest energy pure nanoclusters of silica and titania found previously $32,33,34,36,37,38,49,50,50,51$ used here to calculate mixing energies for our set of titanosilicates. The global minima silica nanoclusters in this size range tend to be quite symmetric and open, and typically possess two or more terminal oxygen defects. Notice that for size $n=24$ we have taken the very low energy fully-coordinated structure reported in ref. 38 structure rather than the reported global minimum structure in ref. 37 which possesses terminal oxygens. In fact, as noted in ref. 38 , these two structures are almost degenerate in energy and the use of the PBEO functional in the present work slightly favours the fully coordinated structure. All pure silica nanocluster structures display both 3- and 4-fold coordinated silicon atoms. Three-fold coordinated silicon atoms are present only when bound to a terminally "double-bonded" oxygen (i.e. formally: $>\mathrm{Si}=\mathrm{O}$, but arguably more accurately: $\left.>\mathrm{Si}^{+}-\mathrm{O}^{-}\right) .53,54$ Such defective terminations are very common in the global minima silica nanoclusters in this size range and are found for all sizes apart from $n=12$ and $n=24$. For $n=12$ the terminal oxygen atoms are bonded to 4-coordinated silicon atoms. In this case they are known as non-bridging oxygen (NBO) atoms where, for each NBO, the nanocluster also possesses a compensating triple coordinated oxygen-site. The combination of these two sites is also known as a 
compensated NBO or a valence alternation pair ${ }^{55,56}$. Titania nanoclusters tend to have relatively more compact and amorphous structures with respect to their silica counterparts. Most of them tend to have two NBO-type terminal oxygen defects. Unlike for the pure silica nanoclusters 3-fold coordinated titanium centres (i.e. $>\mathrm{Ti}=\mathrm{O}$ species) appear to be energetically unstable and the majority of the oxygen-terminated global minima nanoclusters exhibit only NBOs (except for $\left(\mathrm{TiO}_{2}\right)_{2}$ where, due to the extremely small system size, only 3-coordinated Ti centres are possible). The global minima structures for $\left(\mathrm{TiO}_{2}\right)_{10}$ and the largest $\mathrm{n}=24$ titania nanocluster considered are fully coordinated. As for silica this probably indicates a tendency for low energy nanoclusters to exhibit less energetically costly terminal defects with increasing size. ${ }^{38}$ Generally, the respective global minima nanoclusters of $\left(\mathrm{TiO}_{2}\right)_{\mathrm{n}}$ and $\left(\mathrm{SiO}_{2}\right)_{\mathrm{n}}$ are quite distinct, with both systems displaying similar nanocluster structures only for sizes $n=2$ and 12 . 


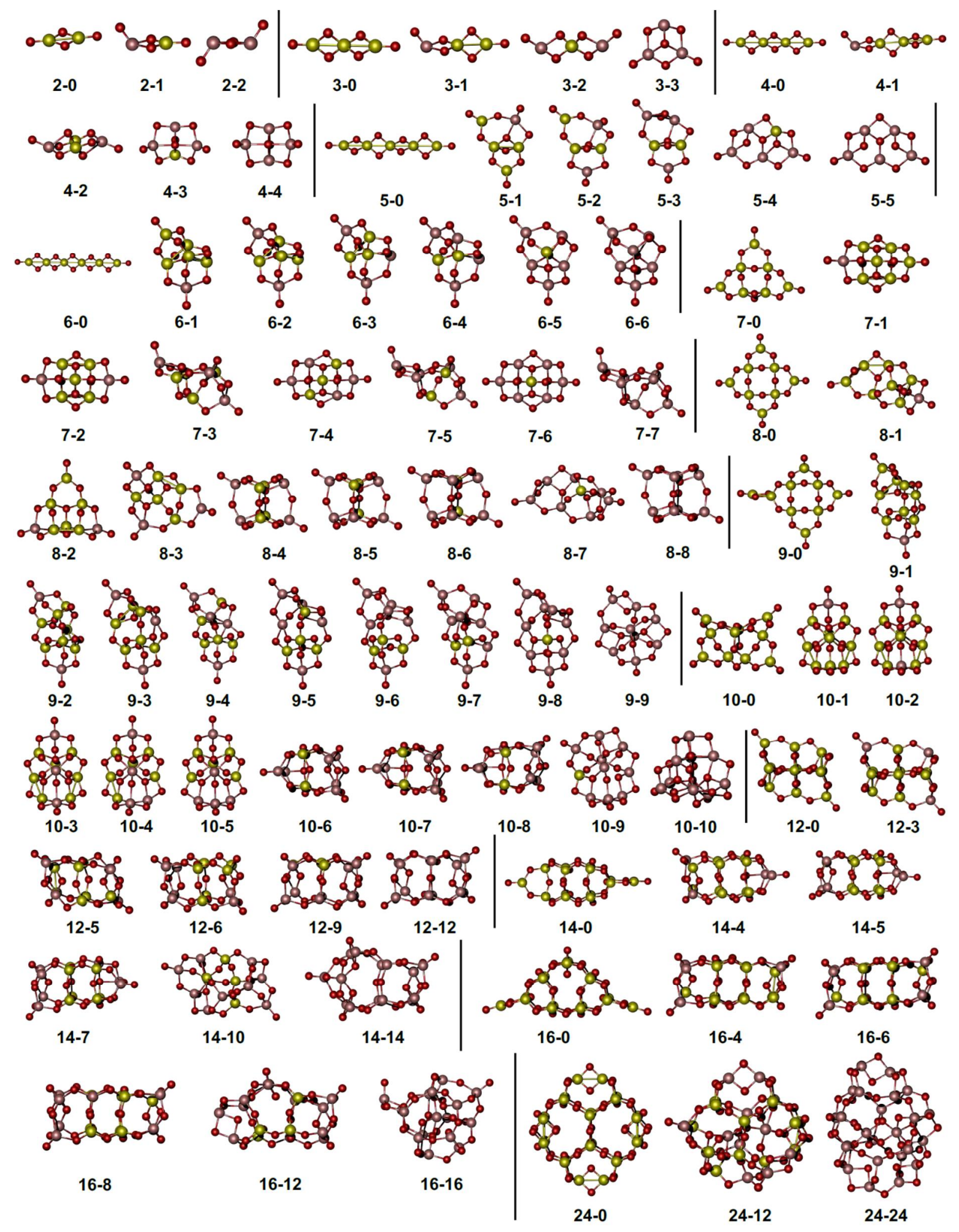

Fig 3. Globally optimized titanosilicate nanoclusters. Pure silica and titania nanoclusters from Fig 2 are also included for completeness. The two numbers assigned for each nanocluster are relative to the total number of units $(n)$ and the number of incorporated $\mathrm{TiO}_{2}$ units, respectively. Structures with sizes $n=2-5$ were previously reported in literature ${ }^{29}$, while all others are new to this study. 
In Fig. 3 we report the lowest energy titanosilicate $\left(\mathrm{Ti}_{x} \mathrm{Si}_{1-x} \mathrm{O}_{2}\right)_{n}$ nanocluster structures found in the present study (i.e. global minima candidates) for a range of different mixing ratios, $\mathrm{x}$, and sizes, $\mathrm{n}$. We note that only structures with sizes $n=2-5$ were previously reported. ${ }^{29}$ Almost all of the titanosilicate nanoclusters present two terminal oxygen defects, mainly of the NBO type at Ti centres (Ti-NBOs). Typically, the titanosilicate nanocluster structures have little or no symmetry unlike the global minima of pure silica nanoclusters. Even at very small $\mathrm{TiO}_{2}$ content, there appear to be a strong influence towards more "titania-like" nanoclusters structures, especially for nanoclusters with sizes $n=5-14$. We note that in several cases there are just a few dominant isomers that cover the whole composition range for a particular system size. The structural similarity of the mixed nanoclusters to those of pure titania can be so strong that in some cases the mixed nanoclusters exhibit the same bonding topology as the respective titania global minimum structure, with only replacements of titanium atoms by silicon atoms (e.g. structures 4-3, 5-4, 6-5, 7-3, 7-5, 8-4, 8-5, 8-6 in Fig. 3). This similarity between mixed $\left(\mathrm{Ti}_{x} \mathrm{Si}_{1-x} \mathrm{O}_{2}\right)_{n}$ nanoclusters and pure $\left(\mathrm{TiO}_{2}\right)_{n}$ might suggest that could be possible to obtain low energy titanosilicate nanoclusters simply by replacing titanium with silicon atoms in pure titania nanoclusters. However, in many cases, we find that the global minima of the mixed nanoclusters have different structures with respect to the pure counterparts (e.g. structures $8-2,8-3,9-1 \cdots 9-6,10-1 \cdots 10-5,10-6 \cdots 10-8$ in Fig. 3:) demonstrating the need for our global optimisation approach.

\section{3) Thermodynamics of mixing}

\section{1) Mixing energy}

As a first approximation, we use total internal electronic energies at OK directly from our DFT based calculations for our evaluations of $\Delta$ of our global optimized nanoclusters and periodic crystalline titanosilicates. In Fig. 4 we plot mixing energies, calculated according equation 3, for all global optimized $\left(\mathrm{Ti}_{x} \mathrm{Si}_{1-x} \mathrm{O}_{2}\right)_{n}$ nanoclusters in Fig. 3 and bulk systems in Fig. 1, as a function of $\mathrm{x}$. We can clearly see in Fig. 4 that the mixing between quartz and rutile to form bulk crystalline titanosilicates is energetically unfavourable (i.e. $\Delta \quad$ values are positive). This result tends to confirm experiments where crystalline titanosilicates have only been successfully synthesised with very small proportions of $\mathrm{TiO}_{2}$ (e.g. TS-1), and otherwise form metastable mixed oxide glasses. However, at the nanoscale, the mixing of pure silica and titania nanoclusters is generally energetically favourable (i.e. $\Delta \quad$ values are generally negative). The nanocluster mixing energy size dependency is also shown in the 3D graph in Fig. 5. Here we use a smoothed continuum surface to represent the discrete set of data points of the mixing energies with respect to $\mathrm{TiO}_{2}$ ratios and nanocluster size. From Figs. 4 and 5 it is clear that for very small nanocluster sizes $(n=1-5)$ the 
mixing energies are quite modest in magnitude $(\leq 0.15 \mathrm{eV}$ ) and can be positive (for $n=3)$ or negative (for $n=4-5$ ). In the latter cases, the most favourable mixing energies are found for $x$ values between $0.5-0.6$. With increasing nanocluster size from $n=6-12$ we observe a rapid increase in the overall energetic favourability of mixing with the most favourable region of mixing occurring for nanoclusters possessing 7, 9, 10 units (i.e. nanoclusters of approximately $1 \mathrm{~nm}$ diameter) and for $\mathrm{x}$ values between $0.4-0.5$. For larger nanocluster sizes the mixing composition for the most favourable mixing is maintained, but the magnitude of $\Delta \quad$ slowly reduces with increasing nanocluster size.

Bulk mixing of $\mathrm{SiO}_{2}$ and $\mathrm{TiO}_{2}$ not energetically favoured $\mathrm{TiO}_{2}$ in clusters energetically favoured
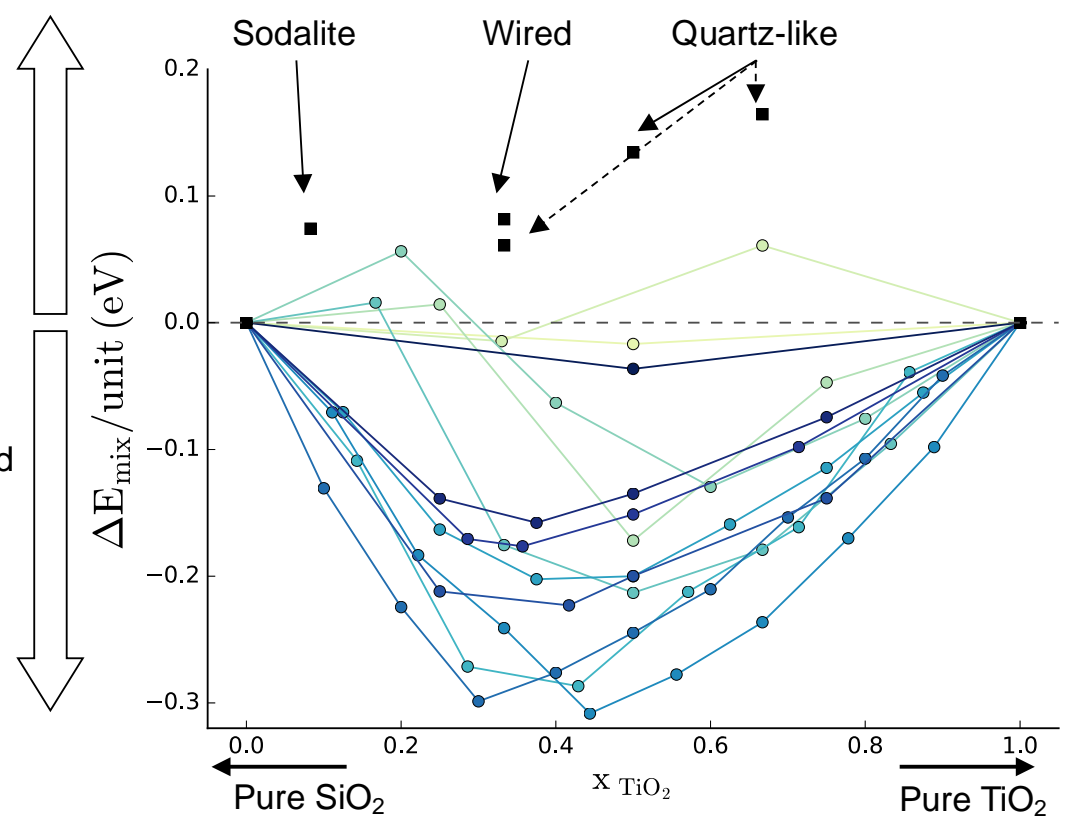

Fig 4. Mixing energy per formula unit versus the $\mathrm{TiO}_{2}$ molar fraction. The black filled squares denote the mixing energies of the bulk periodic structures shown in Fig. 1. The filled coloured circles denote the mixing energies of the globally optimized nanoclusters shown in Fig. 3. Nanocluster sizes are represented by a colour map ranging from light green for smaller nanoclusters to dark blue for larger sizes. 


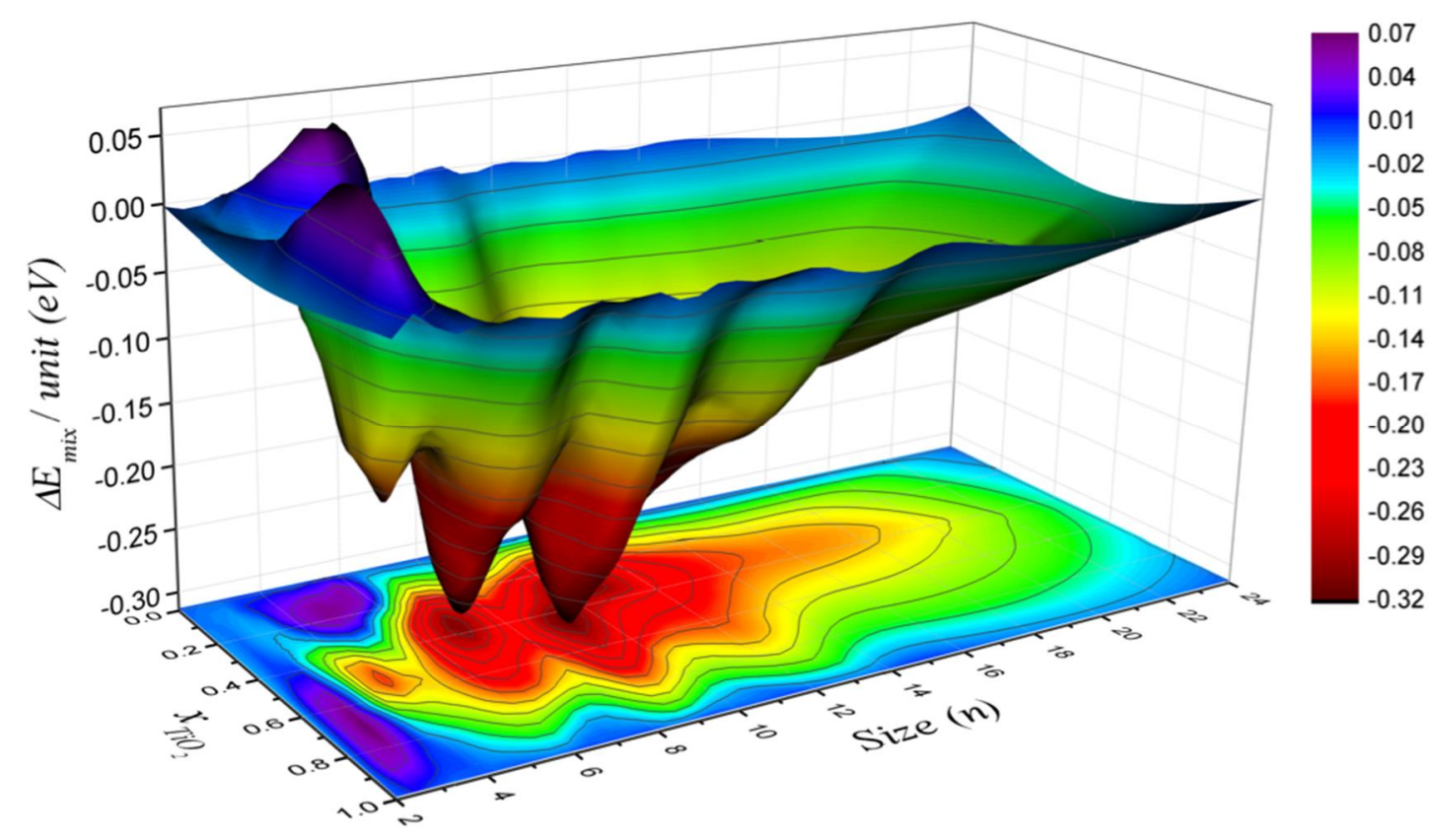

Fig 5. Three-dimensional surface representation of the mixing energy per unit for globally optimized titanosilicates nanoclusters versus the $\mathrm{TiO}_{2}$ molar fraction (x) and system size (n). This 3D surface shows the size dependency of the nanocluster mixing energies reported in the 2D graph in Fig. 4. A 2D contoured projection of the mixing energy map is also shown. The colour map indicating mixing energies ranges from dark blue (energetically unfavourable mixing) to red (energetically favourable mixing). We note that for ease of visualisation the contours and surface have been smoothed by interpolating through their respective underlying sets of discrete data points.

The sign and magnitude of the mixing energies of titanosilicate nanoclusters arises from the competition between several electronic and/or structural factors. For example, the presence of terminating defective oxygen sites and distorted tetrahedral $\mathrm{SiO}_{4}$ groups are both energetically destabilizing factors. In low energy mixed $\left(\mathrm{Ti}_{x} \mathrm{Si}_{1-x} \mathrm{O}_{2}\right)_{n}$ nanoclusters, terminal NBO defects are preferentially found at titanium centres (Ti-NBO) rather than at silicon centres (Si-NBO). This can probably be explained due to the higher ionicity of Ti-O bonds with respect to $\mathrm{Si}-\mathrm{O}$ bonds whereby rupture of the former is relatively less electronically, and thus energetically, destabilizing. Although this tends to favour terminal Ti-NBO groups over Si-NBO groups, Ti centres can also take advantage of internal positions in a nanocluster with high oxygen coordination spheres (making 5-6 Ti-O bonds). The strong tendency of silicon to have directional tetrahedral oxygen coordination means that such positions are not energetically favourable for Si centres. However, taking into account the higher $\mathrm{Si}-\mathrm{O}$ bond strength with respect to $\mathrm{Ti}-\mathrm{O}$ (bond dissociation energies for Ti-O is $666.5 \pm 5.6$ $\mathrm{kJ} / \mathrm{mol}$ while for Si-O it is $799.6 \pm 13.4 \mathrm{~kJ} / \mathrm{mol})^{57} \mathrm{Si}$ centres in tetrahedrally coordinated oxygen environments lower the total nanocluster energy more that Ti centres in the same position. These considerations can inform us as to how mixed nanoclusters can more effectively avoid the energetic 
cost of terminating $\mathrm{NBO}-\mathrm{Si}$ defects and internal $\mathrm{SiO}_{4}$ strain while taking advantage of strong unstrained Si-O bonding and highly coordinated Ti centres by the following set of general structural principles: nanoclusters should possess (i) Ti-NBO rather than Si-NBO terminations, (ii) Ti centres rather than $\mathrm{Si}$ centres in positions having either high oxygen coordination (i.e. >4) or distorted tetrahedrally coordinated oxygen environments, (iii) $\mathrm{Si}$ centres rather than $\mathrm{Ti}$ centres in positions with relatively undistorted tetrahedrally coordinated oxygen environments. Consequently, Si-rich nanoclusters can often be stabilised by following principles (i) and (ii), and Ti-rich nanoclusters may be stabilised by following principle (iii). As a concrete example, for the case of $n=12$ nanoclusters we show in figure 6 how pure and $\left(\mathrm{SiO}_{2}\right)_{12}$ and $\left(\mathrm{TiO}_{2}\right)_{12}$ nanoclusters can be stabilised by following these principles when moving to mixed nanoclusters.

$$
\left(\mathrm{Ti}_{\mathrm{x}} \mathrm{Si}_{1-\mathrm{x}} \mathrm{O}_{2}\right)_{12}
$$

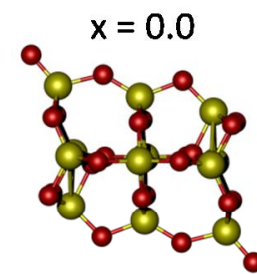

Decreasing mixing energy

NBO-Si centres and strained tetrahedrally coordinated Si centres replaced by $\mathrm{Ti}$, i.e factors (i) and (ii) $x=0.83$

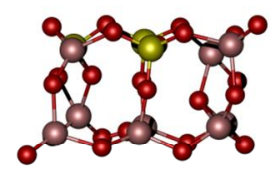

Decreasing mixing energy

Relatively unstrained tetrahedrally coordinated Ti centres replaced by Si, i.e factor (iii).

Fig 6. Example of how negative mixing energy can be rationalised when moving from pure phase $\left(\mathrm{SiO}_{2}\right)_{\mathrm{n}}$ and $\left(\mathrm{TiO}_{2}\right)_{n}$ nanoclusters to mixed nanoclusters for the case of $n=12$ by following principles (i) - (iii) - see end of section 3.1 in main text.

Although we do not have extensive data for sizes higher than 16 units, the set of structures between 2 and 16 units allows for an analysis of some of these factors affecting the stability of mixed systems with size. We suggest that the decrease in mixing energy with increasing system size above 10 units can be largely attributed to the gradual decrease in terminal defects in the pure silica reference nanoclusters (e.g. four for $\left(\mathrm{SiO}_{2}\right)_{10}$, two for $\left(\mathrm{SiO}_{2}\right)_{12},\left(\mathrm{SiO}_{2}\right)_{14}$ and $\left(\mathrm{SiO}_{2}\right)_{16}$ and none for $\left.\left(\mathrm{SiO}_{2}\right)_{24}\right)$. Therefore, titanosilicate nanocluster stabilities are compared with less and less destabilized pure silica systems as the size increases. Finally, following the general trend in Fig. 5, we can predict that the energetic favourability of mixing in titanosilicates nanoclusters with $n>24$ units would rapidly tend to zero. 


\section{2) Mixing free energy}

For a more accurate description of the thermodynamics of $\mathrm{TiO}_{2}-\mathrm{SiO}_{2}$ mixing in titanosilicate nanoclusters we have considered the mixing Gibbs free energies at different temperatures for a set of nanoclusters with selected $\mathrm{TiO}_{2}$ incorporation ratios. Specifically, we evaluate the free energy contributions to the mixing energies at different temperatures and at 1 atmosphere for pure oxide nanoclusters and $50 \%$ mixing for sizes $n=2-16$ units, according to:

$$
\Delta()=\Delta()-\Delta()
$$

where $\Delta$ and $\Delta$ are respectively the enthalpy and entropy of the system including temperature dependent contributions of nanocluster translations $\left(S_{\text {trans }}\right)$, rotations $\left(S_{\text {rot }}\right)$, vibrations $\left(S_{\text {vib }}\right)$ and relevant distinct nanocluster isomer atomic configurations $\left(\mathrm{S}_{\text {conf }}\right)$. Both $\mathrm{S}_{\text {trans }}$ and $\mathrm{S}_{\text {rot }}$ are obtained from standard thermochemical relations and we use calculated harmonic frequencies to evaluate $\mathrm{S}_{\mathrm{vib}}{ }^{58}$. In this work, we neglect the configurational entropy term ( $\left.\mathrm{S}_{\text {conf }}\right)$. This term would take into account the entropic contribution for all the different configurational isomers weighted with respect to their relative energy. Generally, we find that there is large energy separation between (configurational) isomers and global minima for the mixed nanoclusters justifying this assumption. In the Supplementary Information we report the energy separations between our global minima nanoclusters and the respective first few energetically low lying isomers and the probability for each of them to be thermally accessible.

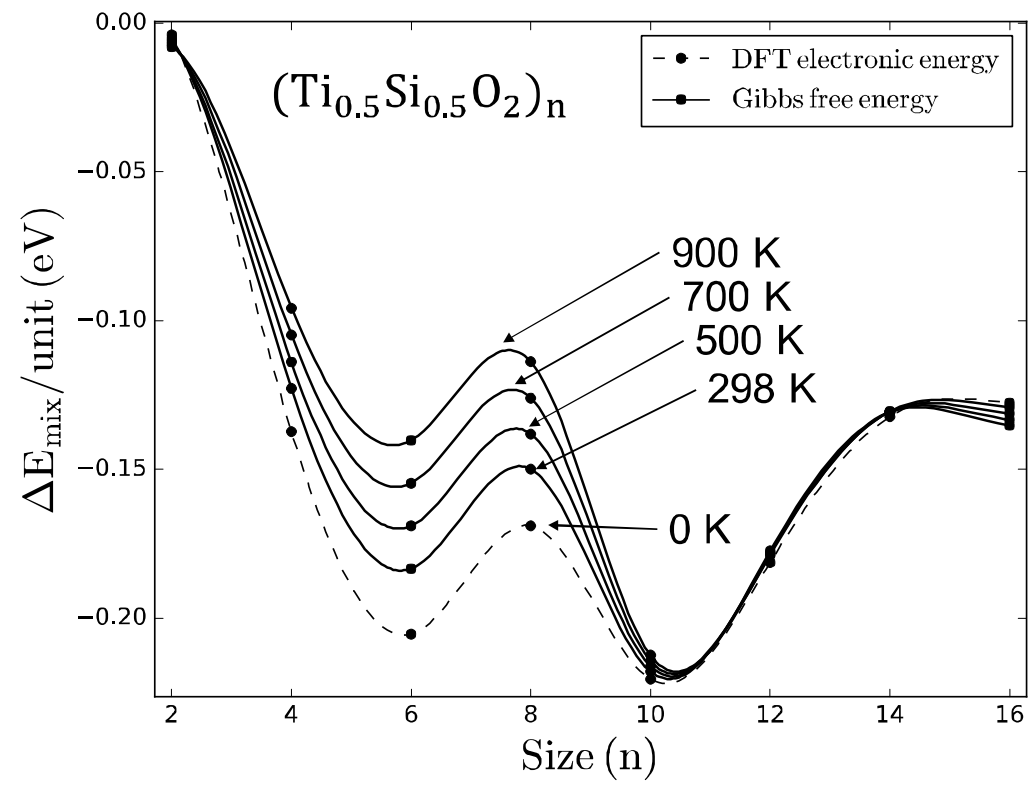

Fig 7. Total mixing electronic energy without zero point correction (dashed line) and Gibbs mixing free energies (solid lines) at different temperatures $(298,500,700$ and $900 \mathrm{~K})$ relative to $\left(\mathrm{Si}_{0.5} \mathrm{Ti}_{0.5} \mathrm{O}_{2}\right)_{\mathrm{n}}$ systems versus the 
nanocluster size $n(n=2,4,6,8,10,12,14$ and 16 units). Energies are plotted on smoothed lines in order to help follow the trends.

In Fig. 7 we plot the mixing free energies at different temperatures $(298,500,700$, and $900 \mathrm{~K})$ calculated according to equation 3 using the calculated Gibbs free energies for each energetic term for the set of nanoclusters mentioned above. Mixing free energies are compared with mixing electronic energies. Overall, the thermodynamic favourability of mixing is found to be conserved with respect to the results for OK when increasing the temperature. Thermal corrections affect mainly nanoclusters with sizes $n=6$ and 8 , but are found to be less relevant for the mixing at other sizes. Generally, thermal corrections lead to slightly less favourable mixing energies for all sizes except for the largest $(n=16)$ nanocluster considered where the thermally corrected mixing free energy is lower than electronic one. A deeper analysis of this effect is out of the scope of the present work, since we are primarily interested in verifying the persistence of favourable mixing in our mixed oxide nanoclusters at finite temperatures.

\section{4) Signatures of mixing}

In an effort to provide experimentally measurable signatures of $\mathrm{TiO}_{2}-\mathrm{SiO}_{2}$ mixing in $\left(\mathrm{Ti}_{x} \mathrm{Si}_{1-x} \mathrm{O}_{2}\right)_{\mathrm{n}}$ nanoclusters, in the following two sections, we analyse calculated harmonic frequencies and average coordination numbers of pure oxide nanoclusters as compared to mixed oxide nanoclusters.

\section{1) Harmonic frequencies}

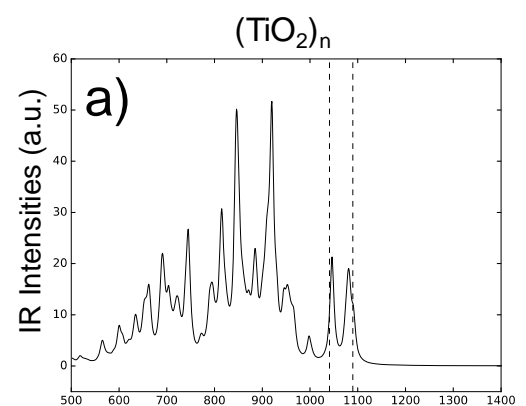

Freq $\left(\mathrm{cm}^{-1}\right)$

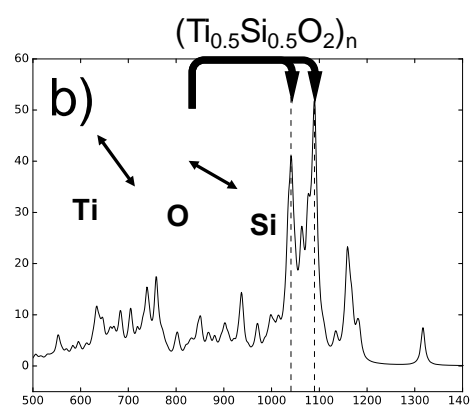

Freq $\left(\mathrm{cm}^{-1}\right)$

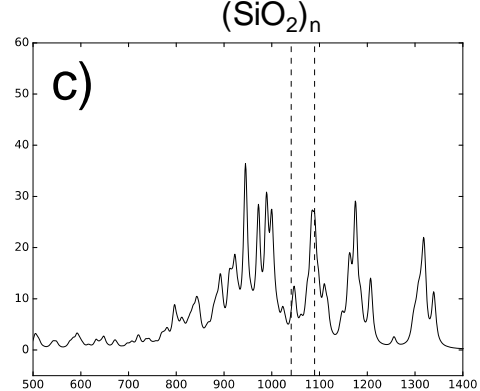

Freq $\left(\mathrm{cm}^{-1}\right)$

Fig 8. Calculated infrared (IR) harmonic vibrational frequencies (in $\mathrm{cm}^{-1}$ ) of pure titania (a), titanosilicate (b) and silica (c) nanoclusters obtained by summing size-scaled IR spectra for nanocluster sizes $n(n=2,4,6,8,10$, 12,14 and 16 units). In b) the anti-symmetric normal mode coupling between Ti-NBO and the vicinal Si-O is shown relative to the most intense peaks. Calculated IR harmonic frequencies for each size are available in the Supplementary Information. All spectra were generated using the Moldraw code ${ }^{59}$ and are made openly digitally available. 
In Fig. 8 we present calculated infrared (IR) harmonic vibrational frequencies for pure titania (Fig 8a), pure silica (Fig 8c) and mixed global optimized (Fig. 8b) nanoclusters. Each spectrum is composed by the sum of size-scaled IR spectra of several $\left(\mathrm{Ti}_{0.5} \mathrm{Si}_{0.5} \mathrm{O}_{2}\right)_{\mathrm{n}}$ nanoclusters (i.e. $=$ $\Sigma^{1}$. ( . ) where is the total IR intensity, 1 is the scaling factor for the ( . ) IR spectrum relative to $\left(\mathrm{Ti}_{0.5} \mathrm{Si}_{0.5} \mathrm{O}_{2}\right)_{\mathrm{n}}$ nanocluster). In other words, the IR spectra shown here are designed to mimic those of hypothetical samples which contain a monotonically decreasing size distribution of nanoclusters. IR spectra for each specific nanocluster size are available in the Supplementary Information. The main feature that we want to highlight from these IR spectra is the presence of two highly intense, distinct and consistent peaks in titanosilicate nanoclusters at around 1035 and $1090 \mathrm{~cm}^{-1}$ (highlighted with the horizontal dashed line in Fig. 7b). These peaks are also present in the pure systems but with significantly lower intensities. In titanosilicate nanoclusters the two peaks are due to couplings between stretching of the Ti-O bond in terminating oxygen defects and the vicinal Si-O bond in the "interior" of the nanocluster (see schematic in in Fig 8b). We note that these two peaks are related to the same normal mode and there is a size dependent redshifting that generates distinct peaks going from $\sim 1090 \mathrm{~cm}^{-1}$ from smaller nanoclusters to $\sim 1035 \mathrm{~cm}^{-1}$ for larger ones.

In pure $\mathrm{TiO}_{2}$ (Fig. 7a) nanoclusters there are two distinct IR peaks that overlap with the ones in titanosilicate nanoclusters. The first peak is slightly blue-shifted at $1046 \mathrm{~cm}^{-1}$ and is due to exclusively to the contribution from the smallest $\left(\mathrm{TiO}_{2}\right)_{2}$ nanocluster. However, we note that the relative intensities of these two peaks are quite low with respect to the most intense peaks. Unlike for titanosilicates, there is also no size dependent red-shifting of these peaks in titania nanoclusters and all peaks around $1090 \mathrm{~cm}^{-1}$ are due to harmonic stretching of terminal Ti-O species. In pure silica (Fig 7c) nanoclusters, there is also an intense peak that coincides with one found for mixed titanosilicate nanoclusters. Here, there is an intense peak at $1085 \mathrm{~cm}^{-1}$ from systems with sizes between 8 and 12 units, due to a complex coupling between stretching and bending modes involving different parts of these nanoclusters.

We suggest that the presence of both vibrational modes at 1090 and $1035 \mathrm{~cm}^{-1}$, particularly enhanced in titanosilicates nanoclusters, can be used as a signature of the presence of mixed $\left(\mathrm{Ti}_{x} \mathrm{Si}_{1}\right.$. $\left.{ }_{\mathrm{x}} \mathrm{O}_{2}\right)_{n}$ nanoclusters 


\section{2) Average Si and Ti coordination numbers}

a)

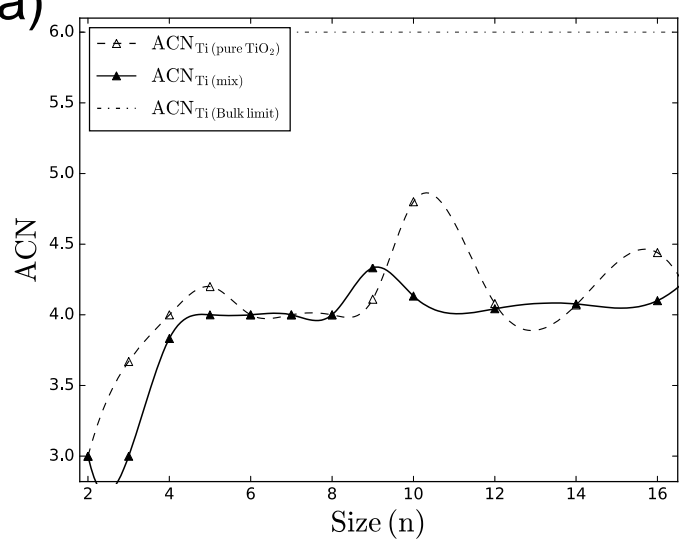

b)

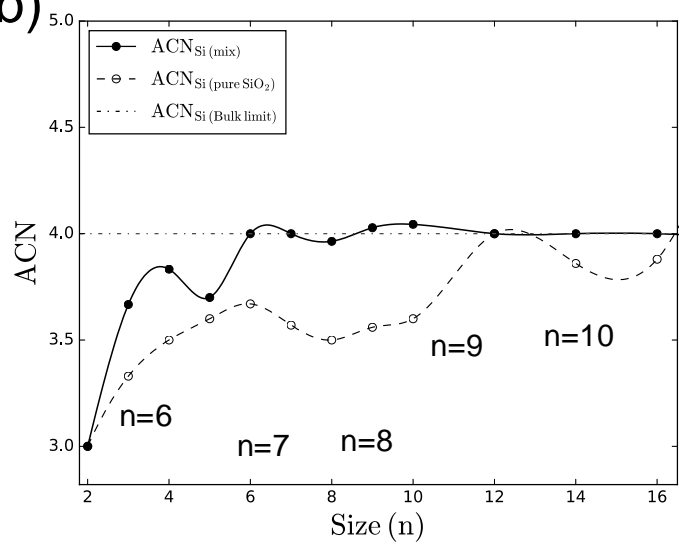

Fig 9. Average cation coordination numbers (ACN) plotted on smoothed lines for easily following trends for: a) titanium centres in pure $\mathrm{TiO}_{2}$ (dashed line) and mixed titanosilicate (solid line) nanoclusters; b) for silicon centres in pure $\mathrm{SiO}_{2}$ (dashed line) and mixed titanosilicate (solid line) nanoclusters. The corresponding oxygen coordination number of the respective cation in the bulk phase is indicated in both cases as a dot-dashed line. All coordination numbers are taken by counting oxygens within a maximum cut-off distance of $2.2 \AA$ from each Ti or Si centre. In mixed systems, for each size, the ACN is averaged over all nanocluster mixing compositions. Figures shown in b) are pure silica structures with $n=6-10$ in order to show the origin of low silicon ACN values for this size regime.

The coordination number of an atom is defined as the number of its nearest neighbours. Average coordination numbers (ACNs) can be experimentally determined by X-Ray techniques such as EXAFS (Extended X-ray Absorption Fine Structure) spectroscopy. Fig. 9 displays the average coordination numbers of titanium (a) and silicon (b) in both pure and mixed systems. In the case of titanium coordination there are no significant differences between pure and mixed systems except for few cases (e.g. for $n=10$ and 16). Here, the ACN for Ti remains between 4 and 4.5 for almost all the size range studied and is well below the bulk value of 6 . In the case of silicon coordination, the ACN in pure silica nanoclusters is persistently lower than in mixed titanosilicate nanoclusters for nearly all sizes. The $\mathrm{ACN}$ for $\mathrm{Si}$ atoms could thus be used as a signature of mixed titanosilicate nanoclusters. The higher value of the $\mathrm{ACN}$ for silicon atoms in titanosilicate nanoclusters is related to the fact that Si atoms can take advantage of mixing with titania to be tetrahedrally 4-coordinated (at the lower energetic cost of producing a terminal Ti-O defect) which is its ideal coordination number (see above discussion). We find that the ACN for Si atoms reaches the ideal bulk value of 4-coordinated at smaller sizes in titanosilicates (6 units) than in pure silica (12-16 units). Indeed, the relative ease with which a bulk-like ACN value for Si centres can be achieved in titanosilicate nanoclusters is one of the driving forces for mixing at smaller nanocluster sizes. 


\section{Conclusions}

In this study we have theoretically investigated the mixing between $\mathrm{TiO}_{2}$ and $\mathrm{SiO}_{2}$ in both bulk phases and in nanoclusters. We employed global optimization to find mixed $\left(\mathrm{Ti}_{x} \mathrm{Si}_{1-x} \mathrm{O}_{2}\right)_{n}$ nanoclusters in a systematic way for a wide range of sizes and mixing compositions. Several new global minima candidates of titanosilicate nanoclusters are thus reported. We observe that bulk mixing is energetically unfavourable which is in agreement with experimental observations. However, the mixing of the two oxides is found to be energetically favourable in the considered nanoclusters from OK up to at least $900 \mathrm{~K}$. The size regime within which mixing is energetically favourable corresponds covers $\left(\mathrm{Ti}_{x} \mathrm{Si}_{1-x} \mathrm{O}_{2}\right)_{n}$ nanoclusters with $\mathrm{n}=2$ - 24 (i.e. to nanoclusters with diameters less than $1.5 \mathrm{~nm}$ ). The maximum mixing favourability is calculated to be when mixed $\left(\mathrm{Ti}_{x} \mathrm{Si}_{1-x} \mathrm{O}_{2}\right)_{n}$ nanoclusters are around $1.0 \mathrm{~nm}$ in diameter (between 9 and 10 units) and when the molar fraction of $\mathrm{TiO}_{2}$ is about 0.3-0.4. We suggest a number of structural-chemical driving forces for mixing in this size range. As a guide for experimentalists to observe and characterize these mixed nano-species we provide two measurable signatures of mixing. Firstly, harmonic frequency calculations show mixed titanosilicate nanoclusters to have a unique and distinct IR fingerprint (intense peaks at 1090 and $1035 \mathrm{~cm}^{-1}$ in IR spectra). Secondly, Si centres in mixed nanoclusters are found to show a higher average coordination relative to pure silica nanoclusters which could, in principle, be observed in EXAFS experiments. We hope that this work will serve as inspiration to experimental researchers to explore the fundamental properties of nanoscale titanosilicate mixed systems in order to synthesize new and highly efficient titanosilicate materials.

\section{Supplementary Information}

We report the coordinates of all global optimized titanosilicate nanoclusters used in this work. We also include a selection of metastable isomers for $\left(\mathrm{Ti}_{0.5} \mathrm{Si}_{0.5} \mathrm{O}_{2}\right)_{n}(n=4,6,8,10,12,14,16)$ and their probabilities to be thermally accessible at different temperatures $(298,500,700,900)$. Calculated harmonic frequencies of the most stable nanoparticles have also been included. Finally we include a table containing total energies and mixing energies for all systems appearing in Fig. 4 and an example of mixing energy normalized by two unit definitions. All structures have also been uploaded in an open access manner in both the NOMAD repository (https://repository.nomad-coe.eu/) and the WASP@N nanocluster database (https://hive.chem.ucl.ac.uk/).

\section{Acknowledgements}

This research was supported by the Spanish MINECO / AEI-FEDER grant: CTQ2015-64618-R and, in part, by Generalitat de Catalunya grants: 2014SGR97 and XRQTC. We acknowledge the Red Española 
de Supercomputación for provision of computing time. We also acknowledge GENCI- CINES/IDRIS (Grant 2016- x2016082131, 2017- x2017082131) and the CCRE-DSI of Université P. M. Curie for computing resources.

\section{References}

\footnotetext{
${ }^{1}$ E.W. McFarland and H. Metiu, Chem. Rev., 2013, 113, 4391-4427.

${ }^{2}$ K. A. Keller, G. Jefferson, and R. J. Kerans, ed. N. P. Bansal, Springer US, Boston, MA, 2005, p. 377.

${ }^{3}$ D. Bergeron, W. A. Castleman, N. O. Jones, and S. N. Khanna, Nano Letters, 2004, 4, 261-265.

${ }^{4}$ K. Fominykh, G. C. Tok, P. Zeller, H. Hajiyani, T. Miller, M. Döblinger, R. Pentcheva, T. Bein, D. Fattakhova-
} Rohlfing, Adv. Funct. Mater., 2017, 27, 1605121.

${ }^{5}$ K.T. Lee, A.A. Lidie, S.Y. Jeon, G.T. Hitz, and S.J. Song, J. Mater. Chem. A, 2013,1, 6199-6207.

${ }^{6}$ R. B. Soriano, J. Wu, and M. G. Kanatzidis, J. Am. Chem. Soc., 2015, 137, 9937-9942.

${ }^{7}$ Taramasso, M., Perego, G., Notari, B. U.S. Patent No. 4410501, 1983.

${ }^{8}$ T.V. Nguyen, H.C. Lee, M. Khan, and O.B. Yang, Sol. Energy, 2007, 81, 529-534.

${ }^{9}$ K. Qi, X. Chen, Y. Liu, J. Xin, C. Mak, and W. Daoud, J. Mater. Chem., 2007, 17, 3504-3508.

${ }^{10}$ S. M. Kuznicki, V. A. Bell, S. Nair, H. W. Hillhouse, R. M. Jacubinas, C. M. Braunbarth, B. H. Toby, and M. Tsapatsis, Nature, 2001, 412, 720-724.

${ }^{11}$ C. Jiang, K. Lee, C. Parlett, M. Bayazit, C. Lau, Q. Ruan, S. Moniz, A. Lee, and J. Tang, Appl. Catal. Gen., 2016, 521, 133-139.

${ }^{12}$ S. Zhan, D. Chen, X. Jiao, and Y. Song, Chem. Commun., 2007, 0, 2043-2045.

${ }^{13}$ P. Cao, G. Zhou, Y. Ren, and H. Xiao, Rsc. Adv., 2016, 6, 6551-6561.

${ }^{14}$ S. M. Kuznicki, U.S. Patent No. 4853202, 1989.

${ }^{15}$ S. M. Kuznicki, K. A. Thrush, F. M. Allen, S. M. Levine, M. M. Hamil, D. T. Hayhurst and M. Mansour, Synth. Micopor. Mater., 1992, 1, 427.

${ }^{16}$ G.S. Henderson and M.E. Fleet, J. Non-Cryst. Solids, 1997, 211, 214-221.

${ }^{17}$ J. S. Rigden, J. K. Walters, P. J. Dirken, M. E. Smith, G. Bushnell-Wye, W. S. Howells and R. J. Newport, J. Phys. Condens. Matter, 1997, 9, 4001.

${ }^{18}$ X. Orignac, H.C. Vasconcelos, and R.M. Almeida, J. Non-Cryst. Solids, 1997, 217, 155-161.

${ }^{19}$ Z. Li, B. Hou, Y. Xu, D. Wu, Y. Sun, W. Hu, and F. Deng, J. Solid. State. Chem., 2005, 178, 1395-1405.

${ }^{20}$ We. Dong, Y. Sun, C. W. Lee, W. Hua, X. Lu, Y. Shi, S. Zhang, J. Chen, and D. Zhao, J. Am. Chem. Soc., 2007, $129,13894-13904$

${ }^{21}$ C. Jiang, K. Y. Lee, C. M. A. Parlett, M. K. Bayazit, C. C. Lau, Q. Ruan, S. J. A. Moniz, A. F. Lee and J. Tang, Appl. Catal. A, 2016, 521, 133-139.

22 S. Plant, L. Cao, F. Yin, Z. Wang, and R. E. Palmer, Nanoscale, 2013, 6, 1258-1263.

${ }^{23}$ S. Plant, L. Cao, and R. E. Palmer, J Am Chem Soc, 2014, 136, 7559-7562. 
${ }^{24}$ R. E. Palmer, L. Cao, and F. Yin, Rev. Sci. Instrum., 2016, 87, 046103.

${ }^{25}$ M. Takeuchi, M. Matsuoka, and H. Yamashita, J Synchrotron Radiat., 2001, 8, 643-644.

${ }^{26}$ S. Bordiga, F. Bonino, A. Damin, C. Lamberti, Phys. Chem. Chem. Phys., 2007, 9, 4854-4878.

${ }^{27}$ M. Landmann, T. Köhler, E. Rauls, T. Frauenheim, and W.G. Schmidt, J. Phys. Condens. Matter, 2014, 26, 253201.

${ }^{28}$ O. Miroshnichenko, S. Posysaev and M. Alatalo, Phys. Chem. Chem. Phys., 2016, 18, 33068-33076.

${ }^{29}$ I. Bandyopadhyay and C. M. Aikens, J. Phys. Chem. A, 2011, 115, 868-879.

${ }^{30}$ D.J. Wales and J.P.K. Doye, J. Phys. Chem. A, 1997, 101, 5111-5116.

${ }^{31}$ http://www-wales.ch.cam.ac.uk/GMIN/

32 L. Tang, L. Linwei, J. Zhao and R. Qiu, J. Comput. Chem., 2011, 33, 163.

${ }^{33}$ M. Y. Chen and D. A. Dixon, J. Chem. Theory Comput., 2013, 9, 3189-3200.

${ }^{34}$ O. Lameil-Garcia, A. Cuko, M. Calatayud, F. Illas, S.T. Bromley, Nanoscale, 2017, 9, 1049-1058.

${ }^{35}$ E. Flikkema and S.T. Bromley, Chem. Phys. Lett., 2003, 378, 622-629.

${ }^{36}$ E. Flikkema and S. T. Bromley, J. Phys. Chem. B, 2004, 108, 9638-9645.

${ }^{37}$ S. T. Bromley and E. Flikkema, Phys. Rev. Lett., 2005, 95, 185505.

${ }^{38}$ E. Flikkema and S. T. Bromley, Phys. Rev. B, 2009, 80, 035402.

${ }^{39}$ M. Matsui and M. Akaogi, Mol. Simul., 1991, 6, 239-244.

${ }^{40}$ V. Blum, R. Gehrke, F. Hanke, P. Havu, V. Havu, X. Ren, K. Reuter and M. Scheffler, Phys. Commun., 2009, 180, 2175-2196.

${ }^{41}$ J. P. Perdew, K. Burke, and M. Ernzerhof, Phys. Rev. Lett., 1996, 77, 3865-3868.

${ }^{42}$ C. Adamo and V. Barone, J. Chem. Phys., 1999, 110, 6158-6169.

${ }^{43}$ S. Hamad, C. R. A. Catlow, S. M. Woodley, S. Lago, J. A. Mejias, J. Phys. Chem. B, 2005, 109, 15741-15748.

${ }^{44}$ S. Bhattacharya, B. H. Sonin, C. J. Jumonville, L. M. Ghiringhelli and N. Marom, Phys. Rev., 2015, 91, 241115

${ }^{45}$ C Brousse, R. C. Newton and O. J. Kleppa, Geochim. Cosmochim. Acta, 1984, 48, 1081-1088.

${ }^{46}$ Gaussian 09, Revision D.01, M. J. Frisch et al., Gaussian, Inc., Wallingford CT, 2013.

${ }^{47}$ A. Schaefer, C. Huber, and R. Ahlrichs, J. Chem. Phys., 1994, 100, 5829-5835.

${ }^{48}$ A. Jain, S. Ong, G. Hautier, W. Chen, W. Richards, S. Dacek, S. Cholia, D. Gunter, D. Skinner, G. Ceder and K. Persson, APL Mater., 2013, 1, 011002.

${ }^{49}$ F. Aguilera-Granja, A. Vega and L. Balbás, J. Chem. Phys., 2016, 144, 234312.

${ }^{50}$ W. C. Lu, C. Z. Wang, V. Nguyen, and M.W. Schmidt, J. Phys. Chem. A, 2003, 107, 6936-6943.

${ }^{51}$ S. M. Woodley, S. Hamad, J. A. Mejias, and C. Catlow, J. Mater. Chem., 2006, 16, 1927-1933.

${ }^{52}$ C. Catlow, S. T. Bromley, S. Hamad, M. Mora-Fonz, A. A. Sokol, S. M. Woodley, Phys. Chem. Chem. Phys., 2010, 12, 786-811.

${ }^{53}$ V.G. Avakyan, V.F. Sidorkin, E.F. Belogolova, S.L. Guselnikov, L.E. Guselnikov, Organometallics, 2006, 25, 6007-6013.

${ }^{54}$ M. A. Zwijnenburg, A. A. Sokol, C. Sousa, S. T. Bromley, J. Chem. Phys., 2009, 131, 034705. 
${ }^{55}$ S. Hamad and S. T. Bromley, Chem. Commun., 2008, 0, 4156-4158.

${ }^{56}$ M. A. Zwijnenburg, F. Illas, J. Chem. Phys. 137 (2012) 154313

${ }^{57}$ Luo, Y.-R. Bond Dissociation Energies. In CRC Handbook of Chemistry and Physics, 89th ed.; Lide, D. R., Ed.;

CRC Press/Taylor and Francis: Boca Raton, FL, 2009

${ }^{58}$ D. A. McQuarrie and J. D. Simons, Molecular Thermodynamics, University Science Book, Sausalito, CA, 1999.

${ }^{59}$ P. Ugliengo, D. Viterbo, G. Chiari, Z. Kristallogr. 1993, 207, 9. 